\title{
Cervical adenoid basal carcinoma associated with invasive squamous cell carcinoma: A report of rare co-existence and review of literature
}

\author{
Boonlert Viriyapak ${ }^{1,2}$, Sung Taek Park², Ah Won Lee ${ }^{3}$, Jong Sup Park², Chung Won Lee ${ }^{2}$, Min Jong Song ${ }^{2}$ and
} SoO Young Hur ${ }^{2 *}$

\begin{abstract}
Cervical adenoid basal carcinoma (ABC) rarely can harbor associated malignancies like adenoid cystic carcinoma or squamous cell carcinoma (SCC), which express markedly different prognosis from a pure ABC, making an appropriate biopsy essential to provide a clear diagnosis and therapeutic plan. We report a 64-year-old asymptomatic lady with an abnormal cervical cytology, who underwent a conization to reveal an $A B C$ with overlying microinvasive SCC. Doubtful resection margins led us to perform radical hysterectomy with lymph node dissection. Subsequent pathological examination showed a true invasive SCC co-existing with ABC, with invasion of the parametrium. Unlike the indolent course of many pure $A B C$ patients, the prognosis of 11 previously reported co-existing invasive SCC with ABC patients appears to depend on the SCC component. Our case reiterates the importance of adequate biopsy with careful interpretation to cover the possibility of a co-existent malignancy. Besides, it presents an argument in favor of radical surgery for the primary treatment of suspicious associated malignancy, and supports adjuvant treatment according to the unfavorable extent of the co-existent invasive carcinoma.
\end{abstract}

Keywords: Adenoid Basal, Squamous Cell, Carcinoma, Cervix Uteri

\section{Background}

Adenoid basal carcinoma $(\mathrm{ABC})$ is a rare cervical malignancy accounting for less than $1 \%$ of all cervical cancers [1]. This tumor closely resembles adenoid cystic carcinoma (ACC), and in fact has been reported as the same entity in some early reports [2], till the first description by Baggish and Woodruff [3] in 1966. The indolent clinical course and an excellent prognosis unique to typical or pure $A B C$ are well recognized. Most patients are asymptomatic, without a detectable cervical mass and are successfully treated by a non-radical surgery with excellent results. In contrast, ACC and basaloid squamous cell carcinoma (BSCC), which morphologically closely resemble $\mathrm{ABC}$, have an aggressive clinical course often associated with recurrence and metastasis [4-6].

\footnotetext{
* Correspondence: hursy@catholic.ac.kr

${ }^{2}$ Department of Obstetrics and Gynecology, Seoul St. Mary's Hospital, The

Catholic University of Korea, Seoul, Republic of Korea

Full list of author information is available at the end of the article
}

This makes accurate distinction between $\mathrm{ABC}$ and its morphological counterparts fairly important.

The association of $A B C$ with other malignancies has been inconstantly reported [4-13]. As the $A B C$ rarely showed malignant behavior, the prognosis appears to depend on the associated malignancy component which also decides the treatment protocol [12]. Although the association of $A B C$ with other malignancies has been reported from time to time, there is paucity of data regarding the specific clinical course, the appropriate diagnostic procedure and the recommended treatment approach.

We report an uncommon association of an invasive squamous cell carcinoma (SCC) with an ABC. The clinical and histological features with the specific immunostains for histogenetic studies are described. Simultaneously, literatures for all reports of these rare co-existent malignancies are reviewed.

\section{(Ciomed Central}




\section{Case presentation}

A 64-year-old Korean female, presented with abnormal cervical cytology screening compatible with "squamous cell carcinoma", no visible cervical lesion was noted on the pelvic examination and the subsequent colposcopy. She has been menopause for 8 years ago and had no history of other gynecologic problem. She therefore underwent a cone biopsy of the cervix with loop electrosurgical excisional procedure (LEEP), which revealed $\mathrm{ABC}$ associated with microinvasive $\mathrm{SCC}$ with the tumors presented at endocervical margin. The remaining cervix was too small to do a repeat cone biopsy, so a pelvic magnetic resonance imaging (MRI) was proposed to find out an occult malignancy. MRI revealed an enhancing mass $(2.0 \times 0.6 \mathrm{~cm})$ involving posterior lip of the cervix with a conclusion of cervical carcinoma (Figure 1). There is no evidence of distant metastasis or any suggestive metastatic lymph node by a positron emission tomography-computed tomography (PET-CT). Clinically stage IB was suggested and following radical hysterectomy, bilateral adnexectomy with pelvic and para-aortic lymph node dissection was undertaken uneventfully (Figure 2A). The pathologic diagnosis was adenoid basal carcinoma co-existing with invasive squamous cell carcinoma. The majority of tumor was $\mathrm{ABC}$ component (about $85 \%$ ) merged with the minor areas of invasive SCC component (about 15\%). There was no evidence of tumor in sections taken from 40 lymph nodes. The removed vagina and all resection margins were clear. Due to deeply infiltration into the stroma and invasion through the right parametrium mostly by the $\mathrm{ABC}$ component, post-operative cisplatin-containing chemoradiation was prescribed to limit the risk of recurrence. After that, she was clinically monitored and stated as no evidence of disease for up to 6 months interval.

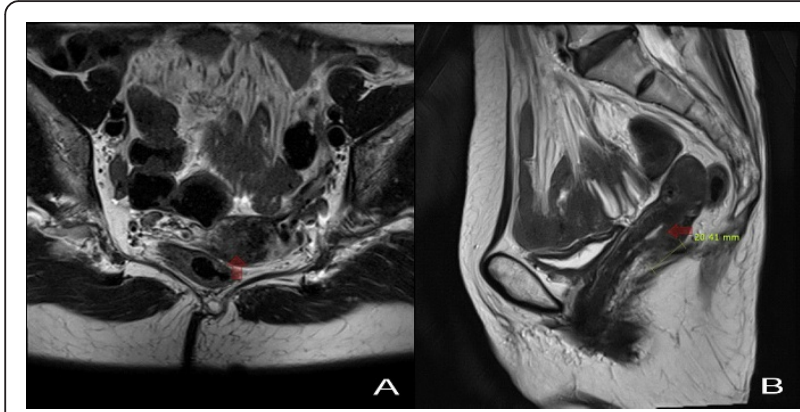

Figure 1 T2-weighted pelvic magnetic resonance images on the axial plane (A) and sagittal plane (B). T2-weighted pelvic magnetic resonance images on the axial plane $(A)$ and sagittal plane (B). Note the cervical mass-like lesion (infiltrative wall thickening with signal enhancement, $2.0 \times 0.6 \mathrm{~cm}$ ) at posterior portion of the uterine cervix with likely posterior vaginal fornix involvement. (arrows point to cervical lesion)

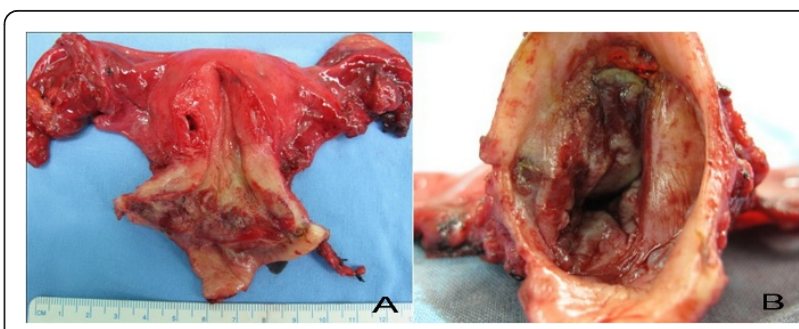

Figure $\mathbf{2}$ Radical hysterectomy. Radical hysterectomy. (A) Whole fresh gross specimen (cervix was opened at $12 \mathrm{o}^{\prime}$ clock position). (B) The cervix, showing ragged avulsion status post conization, no gross definite mass ( $x 1.5$ relatively magnified from Figure $2 \mathrm{~A}$ ).

On pathological examination, the LEEP specimen contained no gross definite lesion. Microscopically, there was diffuse infiltration of small basaloid nests and overlying cervical intraepithelial neoplasia (CIN) grade 3. The CIN lesion showed a focus of microinvasion, compatible with microinvasive SCC. The discrete nests composed of small and uniform basaloid cells with scanty cytoplasm and minimal nuclear atypia. The peripheral palisading morphologic pattern of the tumor nests was typically found and consistent with $\mathrm{ABC}$ lesion. The endocervical resection margin was extensively involved by tumor. The subsequent radical hysterectomy specimen showed ulceration due to previous conization without definite mass formation (Figure 2B). However, a transection of posterior cervix revealed a $2.1 \times 1.8 \times 0.9$ $\mathrm{cm}$ growth infiltrated through the stroma. There was also diffuse infiltration of small basaloid nests without stromal desmoplastic reaction (Figure 3A). Merging with the multi focal areas of $\mathrm{ABC}$ component was the invasive SCC component, which is characterized with irregularly shaped larger nests and occasional central necrosis (Figure 3B). Nuclear atypia and high mitotic figures were noted (Figure $3 \mathrm{C}$ ). Both components showed up to $0.9 \mathrm{~cm}$ stromal invasion within the total $1.2 \mathrm{~cm}$ stromal depth while focally involvement of right parametrium mainly by $\mathrm{ABC}$ was observed. The immunohistochemical stains were parallelly performed. A stain for $\mathrm{p} 63$ and Bcl-2 showed diffuse positive in both $\mathrm{ABC}$ and SCC components (data not shown). While a higher index of Ki-67 staining was observed with squamous cell carcinoma component (40\%) than adenoid basal cell carcinoma component (25\%) (Figure 3D).

\section{Conclusions}

Among different malignancies in the classification of basaloid lesions of the uterine cervix proposed by Grayson and Cooper [14], there was a spectrum of basaloid carcinomas which were stated of histopathologic similarities. Many literatures of $\mathrm{ABC}$ have focused on diagnostic distinction from other mimicked aggressive malignancies 


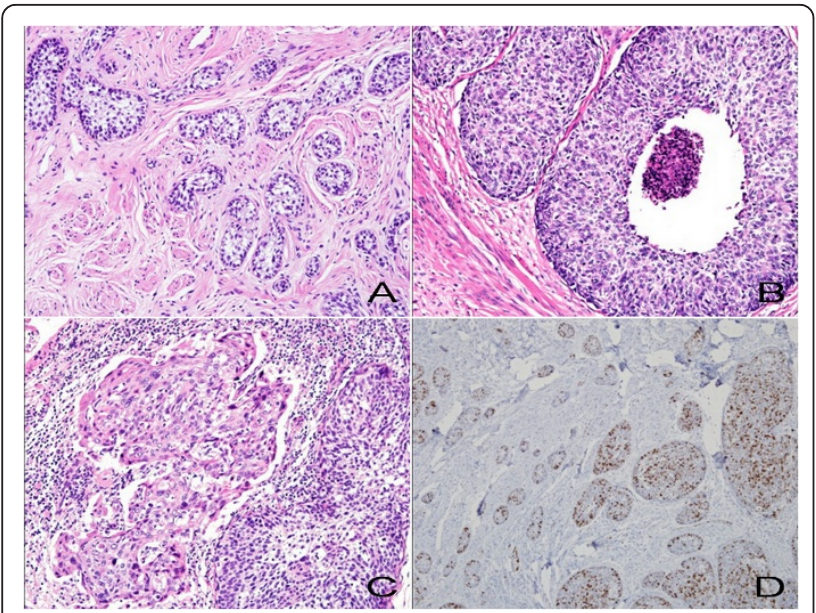

Figure 3 Microscopic findings of the cervical co-existent tumors. Microscopic findings of the cervical co-existent tumors. (A) The cervical stroma with infiltrated small basaloid nests of adenoid basal carcinoma component without stromal desmoplastic reaction (hematoxylin and eosin, $\times 200$ ). (B) The admixed larger nests of squamous cell carcinoma component with central necrosis, and (C) severe nuclear atypia (hematoxylin and eosin, $\times 200$ ). (D) The Ki-67 immunohistochemical stain, showing a higher index of staining in squamous cell carcinoma component than adenoid basal carcinoma component $(\times 200)$

such as ACC, BSCC and small cell (neuroendocrine) carcinoma and to elucidate its histopathogenesis. Meanwhile some reports also revealed valid evidences of the associated malignancy within morphologically pure $A B C$ [4-17]. Since this association was initially documented in 1971 [7], most of the associated malignancies have been reported mixedly with pure $\mathrm{ABCs}$ in the early literatures. The concept of co-existence was then clarified by the divergent epithelial differentiation of the $\mathrm{ABC}$ lesion producing associated squamous, adenosquamous or rarely adenomatous malignant components which support the histogenetic pluripotent reserve cell origin of $A B C$ [9]. The co-existing $\mathrm{ABC}$ with occasionally identified transitional areas to other malignancy mainly ACC, also showing the potential precursor concept [5,9]. Additionally, an associated malignancy could occur by the co-incidence of transformed tumor by the common pathogenetic highrisk human papillomavirus (HPV) integration in both components [10-12]. Although the precise frequency of these rare co-existences is hampered by the selection bias on the case-report evidences, it has shown a substantial account [18] and thus stresses the significance of careful approach with a suspect of associating malignancy in any provisional diagnosed $\mathrm{ABC}$.

Recently, including our case, there are total 98 reported morphologically diagnosed ABCs [1-19]. Apart from the pure lesions, there are 27 cases $(27.5 \%)$ described by $A B C$ associated with other invasive malignancy in a single neoplasm. Regarding to this review, we firstly determine the common clinicopathological features of these specified cervical co-existent tumors which were sequentially summarized in the Additional File 1 table 1 . Most of the patients were menopausal non-Caucasian women. The mean age was 66.7 years (44-84). The most frequent co-existing histology was SCC which accounts for 12 cases, ACC as second most found was co-existed in 9 cases while only 2 associated adenocarcinomas had been specified.

Typically, pure $\mathrm{ABCs}$ are asymptomatic, frequently presented by abnormal Pap smear which often associated with high grade squamous intraepithelial lesions (> 90\%) $[5,18]$. These patients have been successfully treated by non-radical surgery such as simple hysterectomy without documented recurrence or tumor-related death. While if associated with other malignancy, 11 (52.6\%) in 19 assessable presentations were asymptomatic with abnormal cervical cytology and most of these had no gross cervical lesion, implying that an associated invasive carcinoma is possibly presented by inactive clinical features. Two patients with co-existent malignancy including our case reported malignant cytology which less likely occurred in the pure ABC group. Moreover, even on comprehensive colposcopy, unusual ulcerative lesion of $\mathrm{ABC}$ or other infiltrative malignancy may not be detected. Thus, a cone biopsy is recommended to cover the most represented site of entire cervical tumors [20]. It was recorded that this procedure yielded a high definite diagnosis rate for the co-existent malignancy especially for these asymptomatic undetectable mass patients (6 in 9 patients, 66.6\%). Comparing this data, the reviewed co-existent cases with a cervical mass at the presenting time showed its poorer outcome. It would be explained by undiagnosed associated SCC component resulted from an only cervical biopsy and inadequate treatment course for the real status of the associated invasive carcinoma.

In the LEEP specimen, only a focus of microinvasive SCC overlying multiple small nests of $A B C$ was diagnosed. Suggesting that theoretically, area of true invasive carcinoma may not represent in all tumor area or generally admixed with $\mathrm{ABC}$ component, but can produce a distinct infiltrate through the deep part of stroma $[1,12]$ and that resulted in possibility of inadequate assessment by an excisional biopsy. The endocervical resection margin which markedly positive mostly by $\mathrm{ABC}$ indicated that we could not exclude a hiding admixed ACC or a further invasive SCC accompanied in the remaining cervix and a repeat conization would be recommended [21]. Considering small residual cervix in the after-conization menopausal patient, a re-conization may be at risk of accidental injury to bladder or rectum with remarkable bleeding or subsequent infection [20]. High 
accuracy imaging to detect cervical tumor or local invasion by MRI (77-90\% accuracy) [22] was a reasonable option and was again proved by our case. Also with concern on a risk of para-aortic lymph node spreading at a rate of $2-4 \%$ for this clinical stage IB1 cervical cancer [23], we additionally performed para-aortic lymph node dissection at the time of radical hysterectomy, bilateral salpingooophorectomy and pelvic lymph node dissection. The final revealed co-existence compiling $A B C$ associated with truly invasive SCC supported our idea to treat this initially indefinite invasive diagnosed patient as a patient with locally invasive malignancy instead of doing simple hysterectomy which is described as the recommended operation for a typical $A B C$ or even with microinvasive SCC.

The pathological diagnosis was obtained by a matching with the described morphologic criteria based on the current World Health Organization histological classification of tumors of the uterine cervix [1]. The infiltrating foci of the $\mathrm{ABC}$ component showed typical small uniform basaloid cell nest without desmoplastic stromal reaction. Squamous differentiation was diffuse and more frequently detected at the superficial area and focal area adjacent to the SCC component, forming cellular transition to the associated carcinoma $[4,9]$. These histomorphological findings were clearly distinct the $\mathrm{ABC}$ component from the other tumors with basaloid feature like ACC and BSCC which commonly presented with high nuclear pleomorphism, mitotic figure and cribriform or solid growth pattern [14]. Occupying at the most central part of tumors was the invasive squamous component which was deeply infiltrating tumor composed of larger nests of pleomorphic large, non-keratinized squamous differentiated cells with severe nuclear atypia and frequent mitotic features. Obviously this tumor presented a high growth appearance and highly invasive pattern by showing central necrosis and extensive stromal desmoplastic reaction respectively [1]. The result of p63 stain was typical in our case [17] which showed diffuse expression in both $\mathrm{ABC}$ and SCC components intensively by the palisading basaloid cells and suprabasal squamous differentiated cells in the central area of the superficial nests, while markedly reduced reaction in focal glandular (adenoid) differentiated area. The selective Bcl-2 positive reaction in basaloid tumor and SCC shown in our sections helpfully confirmed the basaloid squamous histogenesis and supported their neoplastic natures by showing anti-apoptotic genetic alteration in both components [18]. Moreover, for the cell cycle activity correlated Ki-67 stain which generally reduced expression in the basaloid and adenoid area $[16,17]$, it was similar to our low index for $\mathrm{ABC}$ component and different to a well-defined stronger Ki-67 expression by SCC component. This was implied to higher proliferative activity and indicated distinct malignant potential of the invasive SCC, which corresponded to the central necrosis finding observed on the morphologic exam.

Likewise report of pure $\mathrm{ABCs}$, almost all patients in co-existence group were clinically stage I which primarily treated by a type of hysterectomy. Along with 19 sufficient treatment data, the specific treatments were prescribed mostly depend on nature and stage of the revealed associated invasive malignancy either before or after primary surgery. Obviously different from the pure $\mathrm{ABCs}$, only 3 co-existent cases (15.7\%) have been treated by exclusively non-radical hysterectomy. Postoperative adjuvant therapies mainly by radiation were given to 5 cases $(26.3 \%)$ with 2 of these had received adjuvant treatment after inadequate simple hysterectomy. This data would suggest that a primary radical treatment according to the extension of associated malignancy formally by radical hysterectomy with lymphadenectomy [23] should be recommended and may prevent unnecessary exposures to radiotherapy and/or chemotherapy.

Including our case with 6 months disease-free interval after postoperative chemoradiaton, the reviewed treatment outcomes of the $\mathrm{ABC}$ with associated malignancy patients had mean follow-up time of 31.1 months (0120) with no evidence of disease in overall 13 of 20 retrievable cases (65.0\%). Unlike the favorable outcome of pure $\mathrm{ABCs}$, recurrences were found in 4 cases (20.0\%) and 3 of these died of disease.

An invasive malignancy may simultaneously associated with any cervical $\mathrm{ABC}$, that need an adequate diagnostic cone biopsy which can provide clear diagnosis and would serves both needs of proper radical therapy if a likely $A B C$ with co-existent malignancy is diagnosed and also to prevent the over treatment of a pure ABC. Occasionally, if indefinite tissue diagnosis, an acceptable diagnostic option like MRI may be in preference. Through a decision with suspicious occult invasive carcinoma, the primary radical surgery should be considered. In advance, proper adjuvant treatment such as post-operative radiotherapy would be suggested according to the extent of the associated invasive malignancy.

\section{Consent}

Written informed consent was obtained from the patient for publication of this case report and any accompanying images. A copy of the written consent is available for review by the Editor-in-Chief of this journal. 


\section{Additional material}

Additional file 1: Table 1. Literature summary of reported cases of diagnosed adenoid basal carcinoma with associated invasive malignancy of the uterine cervix

\section{Acknowledgements}

We thank our oncologic nurses(Kyung Suk Min \& Su Min Jung) and pathologic technicians for many kind helps throughout the specimen and pathological processes

\section{Author details}

'Department of Obstetrics and Gynecology, Faculty of Medicine Siriraj Hospital, Mahidol University, Bangkok, Thailand. ${ }^{2}$ Department of Obstetrics and Gynecology, Seoul St. Mary's Hospital, The Catholic University of Korea, Seoul, Republic of Korea. ${ }^{3}$ Department of Pathology, Seoul St. Mary's Hospital, The Catholic University of Korea, Seoul, Republic of Korea.

\section{Authors' contributions}

BV contributed mainly in designing, literature review and writing work. SYH, the corresponding author who provided the case, planed and approved the written work. STP helped in correction of the manuscript. AWL performed the histopathologic process and described all findings and JSP gave advices and edited the discussion. CWL and MJS worked on the clinical presentation. All authors read and approved the final manuscript.

\section{Competing interests}

The authors declare that they have no competing interests.

Received: 20 June 2011 Accepted: 18 October 2011

Published: 18 October 2011

\section{References}

1. Wells M, Ostor AG, Crum CP, Franceschi S, Tommasino M, Nesland JM Goodman AK, Sankaranarayanan R, Hanselaar AG, Albores-Saavedra J: Epithelial Tumours. In Pathology and Genetics of Tumours of the Breast and female genital organs. Volume 5. Edited by: Tavassoli FA, Devilee P. Lyon: IARC Press; 2003:262-286, [World Health Organization Classification of Tumours,].

2. Tchertkoff V, Sedlis A: Cylindroma of the cervix. Am J Obstet Gynecol 1962, 84:749-752.

3. Baggish MS, Woodruff JD: Adenoid-basal carcinoma of the cervix. Obstet Gynecol 1966, 28:213-218.

4. Brainard JA, Hart WR: Adenoid basal epitheliomas of the uterine cervix: a reevaluation of distinctive cervical basaloid lesions currently classified as adenoid basal carcinoma and adenoid basal hyperplasia. Am J Surg Pathol 1998, 22:965-975.

5. Ferry JA, Scully RE: "Adenoid cystic" carcinoma and adenoid basal carcinoma of the uterine cervix. A study of 28 cases. Am J Surg Pathol 1988, 12:134-144.

6. Daroca PJ Jr, Dhurandhar HN: Basaloid carcinoma of uterine cervix. Am J Surg Pathol 1980, 4:235-239.

7. Baggish MS, Woodruff JD: Adenoid basal lesions of the cervix. Obstet Gynecol 1971, 37:807-819.

8. Clement PB, Zubovits JT, Young RH, Scully RE: Malignant mullerian mixed tumors of the uterine cervix: a report of nine cases of a neoplasm with morphology often different from its counterpart in the corpus. Int J Gynecol Pathol 1998, 17:211-222.

9. Grayson W, Taylor LF, Cooper K: Adenoid cystic and adenoid basal carcinoma of the uterine cervix: comparative morphologic, mucin, and immunohistochemical profile of two rare neoplasms of putative 'reserve cell' origin. Am J Surg Pathol 1999, 23:448-458

10. Grayson W, Taylor LF, Cooper K: Carcinosarcoma of the uterine cervix: a report of eight cases with immunohistochemical analysis and evaluation of human papillomavirus status. Am J Surg Pathol 2001, 25:338-347.

11. Takeshima Y, Amatya VJ, Nakayori F, Nakano T, Iwaoki Y, Daitoku K, Inai K: Co-existent carcinosarcoma and adenoid basal carcinoma of the uterine cervix and correlation with human papillomavirus infection. Int J Gynecol Pathol 2002, 21:186-190.

12. Parwani AV, Smith Sehdev AE, Kurman RJ, Ronnett B: Cervical adenoid basal tumors comprised of adenoid basal epithelioma associated with various types of invasive carcinoma: clinicopathologic features, human papillomavirus DNA detection, and P16 expression. Hum Pathol 2005, 36:82-90.

13. Teramoto N, Nishimura R, Saeki T, Nogawa T, Hiura M: Adenoid basal carcinoma of the uterine cervix: report of two cases with reference to adenosquamous carcinoma. Pathol Int 2005, 55:445-452.

14. Grayson W, Cooper K: A reappraisal of "basaloid carcinoma" of the cervix, and the differential diagnosis of basaloid cervical neoplasms. Adv Anat Pathol 2002, 9:290-300.

15. Jones MW, Kounelis S, Papadaki H, Bakker A, Swalsky PA, Finkelstein SD: The origin and molecular characterization of adenoid basal carcinoma of the uterine cervix. Int J Gynecol Pathol 1997, 16:301-306.

16. Senzaki H, Osaki T, Uemura Y, Kiyozuka Y, Ogura E, Okamura A, Tsubura A: Adenoid basal carcinoma of the uterine cervix: immunohistochemical study and literature review. Jpn J Clin Oncol 1997, 27:437-441.

17. Cviko A, Briem B, Granter SR, Pinto AP, Wang TY, Yang YC, Chen BF, Yang A Sheets EE, McKeon FD, Crum CP: Adenoid basal carcinomas of the cervix: a unique morphological evolution with cell cycle correlates. Hum Pathol 2000, 31:740-744.

18. Russell MJ, Fadare O: Adenoid basal lesions of the uterine cervix: evolving terminology and clinicopathological concepts. Diagn Pathol 2006, 1:18

19. Kuroda N, Hirano K, Ohara M, Hirouchi T, Mizuno K, Kubo A, Enzan H: Adenoid basal carcinoma arising in the cervical polyp: an immunohistochemical study of stromal cells. Med Mol Morphol 2007, 40:112-114.

20. Lindeque BG: Management of cervical premalignant lesions. Best Pract Res Clin Obstet Gynaecol 2005, 19:545-561.

21. Kim YJ, Lee WM, Lee JY, Cho SH, Park MI, Kim KT: Adenoid basal carcinoma of the uterine cervix: Reports of two cases. J Women Med 2009, 2:132-136.

22. Zand KR, Reinhold C, Abe H, Maheshwari S, Mohamed A, Upegui D: Magnetic resonance imaging of the cervix. Cancer Imaging 2007, 7:69-76

23. Sakuragi N: Up-to-date management of lymph node metastasis and the role of tailored lymphadenectomy in cervical cancer. Int J Clin Oncol 2007, 12:165-175.

doi:10.1186/1477-7819-9-132

Cite this article as: Viriyapak et al:: Cervical adenoid basal carcinoma associated with invasive squamous cell carcinoma: A report of rare coexistence and review of literature. World Journal of Surgical Oncology 2011 9:132

\section{Submit your next manuscript to BioMed Central and take full advantage of:}

- Convenient online submission

- Thorough peer review

- No space constraints or color figure charges

- Immediate publication on acceptance

- Inclusion in PubMed, CAS, Scopus and Google Scholar

- Research which is freely available for redistribution 